\title{
Hemangioma cavernoso laríngeo en adultos: Reporte de un caso y revisión de la literatura
}

\section{Cavernous hemangioma of the larynx in adults: Case report and literature review}

Felipe Gauna P1, Patricia Moreno S', Agustín Abarca $\mathbf{S}^{2}$, Miguel Cancino $\mathbf{C}^{2}$, Rodrigo Arregui $\mathbf{V}^{2}$.

\begin{abstract}
RESUMEN
Los hemangiomas son uno de los tumores más frecuentes en cabeza y cuello. Los hemangiomas de laringe se pueden dividir clínicamente en formas infantiles y adultas. En niños es una patología frecuente, mientras que en adultos es muy infrecuente. En adultos, tiene mayor incidencia en hombres y su ubicación más frecuente es supraglótica. Su histología más frecuente es el hemangioma cavernoso $(\mathrm{HC})$, que corresponde a una malformación de origen venoso, con paredes delgadas y vasos dilatados. La presentación más habitual es con disfonía o ronquera de meses o años de evolución y se sospecha mediante la nasofaringolaringoscopía o el estudio de imágenes (TC y/o RM). Existen distintas formas de tratamiento, incluidas la observación, la corticoterapia, la radioterapia, la cirugía láser, la cirugía abierta, etc., sin existir un consenso sobre la mejor opción.

Se presenta el caso de un paciente de 43 años con antecedentes de tabaquismo que consultó por una historia de disfonía de un año de evolución. Se diagnosticó mediante nasofaringolaringoscopía y TC de cuello una gran masa supraglótica que se extirpó mediante un abordaje de laringofisura. La biopsia diferida informó un HC.
\end{abstract}

Palabras clave: Hemangioma cavernoso, laringe, adulto, disfonía.

\begin{abstract}
Hemangiomas are one of the most frequent head and neck tumors. Clinically, they can be divided into childish and adult forms. In children it is a frequent pathology, whereas in adults it is very rare. In adults, it has a higher incidence in men and its more frequent location is supraglottic. Its most frequent histology is the cavernous hemangioma, which corresponds to a malformation of venous origin, with thin walls and dilated vessels. The most common presentation is with the disphony or hoarseness lasting from months to years of evolution and suspicion is made through nasofibroscopy or the imaging study (CT and / or MRI). There are different forms of treatment, including observation, corticotherapy, radiotherapy, laser surgery, open surgery, etc., without a consensus on the best option.
\end{abstract}

1 Interno de Medicina, Universidad de Chile.

2 Médico del Servicio de Otorrinolaringología, Hospital Barros Luco Trudeau. Universidad de Chile. 
We present the case of a 43 year old male with a history of smoking who consulted for dysphonia of a year of evolution. A large supraglottic mass was diagnosed through nasofibroscopy and CT of the neck, which is removed by a laryngopharyngeal approach. Deferred biopsy reported a cavernous hemangioma.

Key words: Cavernous hemangioma, larynx, adult, disphony, hoarseness.

\section{INTRODUCCION}

Los hemangiomas corresponden a uno de los tumores más frecuentes en cabeza y cuello. El $65 \%$ del total de hemangiomas se localizan en cabeza y cuello, siendo los sitios más frecuentes la glándula parótida, la lengua y la laringe en niños ${ }^{1,2}$. Los hemangiomas de laringe se pueden dividir en 2 tipos: infantiles, donde representa una patología frecuente, que alcanza $4 \%-5 \%$ de incidencia; y una forma adulta, donde es poco común, por lo que se desconoce su real incidencia ${ }^{1-6}$.

El hemangioma cavernoso ( $\mathrm{HC}$ ) corresponde a una malformación venosa y se caracteriza por formar ovillos de vasos de pared delgada, a veces dilatados, en los que puede haber sangre estancada, lo que predispone a la formación de trombosis ${ }^{7}$. Cuando se logra observar de forma directa o mediante nasofaringolaringoscopía, es descrita como una lesión con coloración azula$\mathrm{da}^{2,8-10}$. En adultos, es más frecuente en hombres y en la región glótica y supraglótica, a diferencia de Ios niños, donde es más frecuente en mujeres y en región subglótica ${ }^{1,3-5}$.

En los casos reportados de HC laríngeo en adultos, la presentación más habitual es en hombres alrededor de los 40-50 años con disfonía 0 ronquera persistente de varios meses de evolución, que aumenta de forma progresiva, sin factores de

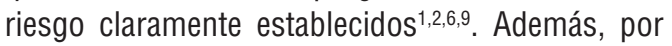
el sitio donde se ubican con mayor frecuencia las lesiones también se pueden presentar con disfagia, estridor y disnea ${ }^{4,7}$. Estos síntomas pueden estar presentes por varios años antes de llevar a consultar ${ }^{3}$.

El diagnóstico de estas lesiones se puede apoyar en las imágenes: tomografía computarizada (TC), resonancia magnética (RM) o ecotomografía doppler ${ }^{8}$, aunque a menudo el estudio se inicia con una nasofaringolaringoscopía donde se pueden observar características que orientan a una lesión de origen vascular².
Se han utilizado distintas opciones de tratamiento, tales como corticoides sistémicos, corticoides o alcohol intralesionales, ablación con láser, interferón, microdebridador, radioterapia criocirugía 0 cirugía abierta ${ }^{1,2,5,9,11}$. No existe un procedimiento quirúrgico óptimo universalmente aceptado por lo infrecuente de la patología y la falta de series de casos $^{5}$.

\section{CASO CLINICO}

Se presenta el caso de un hombre de 43 años, con antecedentes de tabaquismo con un índice de consumo de paquetes por año (IPA) de 3, con una historia de 1 año de evolución de disfonía progresiva sin variación diaria asociada a disfagia durante los últimos 3 meses. Sin baja de peso.

Consultó de forma ambulatoria donde se solicitó una tomografía computarizada de cuello con contraste que mostraba una lesión sólida oval en espacio paralaríngeo y preepiglótico derecho de $5,5 \times 3 \times 4 \mathrm{cms}$. con refuerzo heterogéneo (Figura 1).

Se realizó una nasofaringolaringoscopía que mostraba una masa a nivel hipofaríngeo lobulada de superficie lisa.

Fue derivado al Servicio de Otorrinolaringología del Hospital Barros Luco Trudeau. Se presentó con persistencia de la sintomatología. Al examen físico no se encontraron hallazgos y el paciente se encontraba sin disnea. Se decidió realizar nueva nasofaringolaringoscopía que mostraba una gran masa de superficie homogénea en receso aritenoepiglótico derecho, con mucosa sana, parcialmente obstructiva (Figura 2).

Bajo el diagnóstico de un tumor laríngeo supraglótico probablemente benigno se decidió realizar su resección quirúrgica mediante laringofisura más traqueostomía. Se realizó el procedimiento accediendo a la laringe por línea media observándose el tumor que se disecó respetando la mucosa 


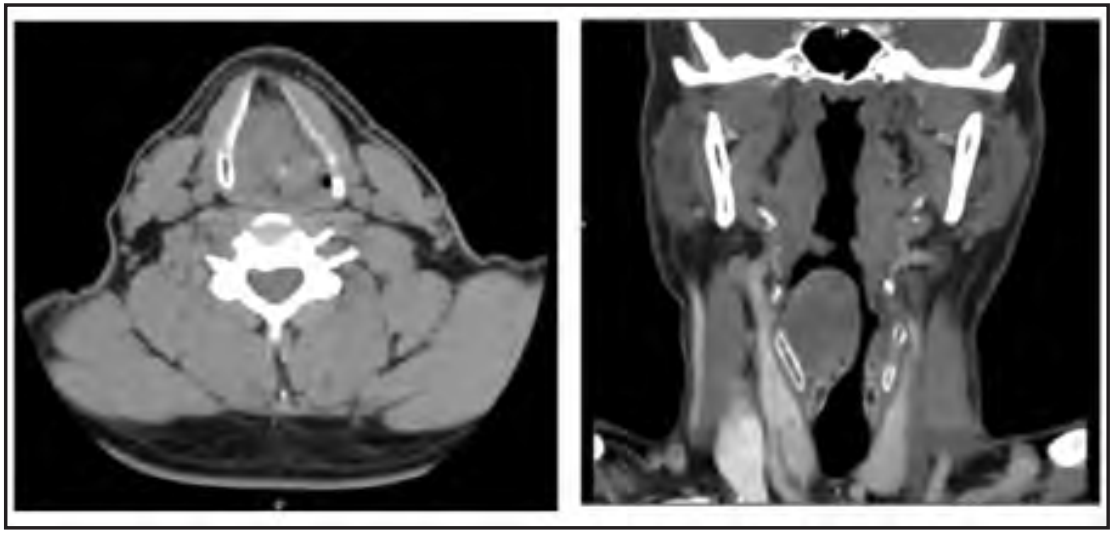

Figura 1. Tomografía computarizada de cuello con contraste. A la izquierda corte axial y a la derecha corte coronal. Se observa una gran masa oval supraglótica derecha parcialmente obstructiva.

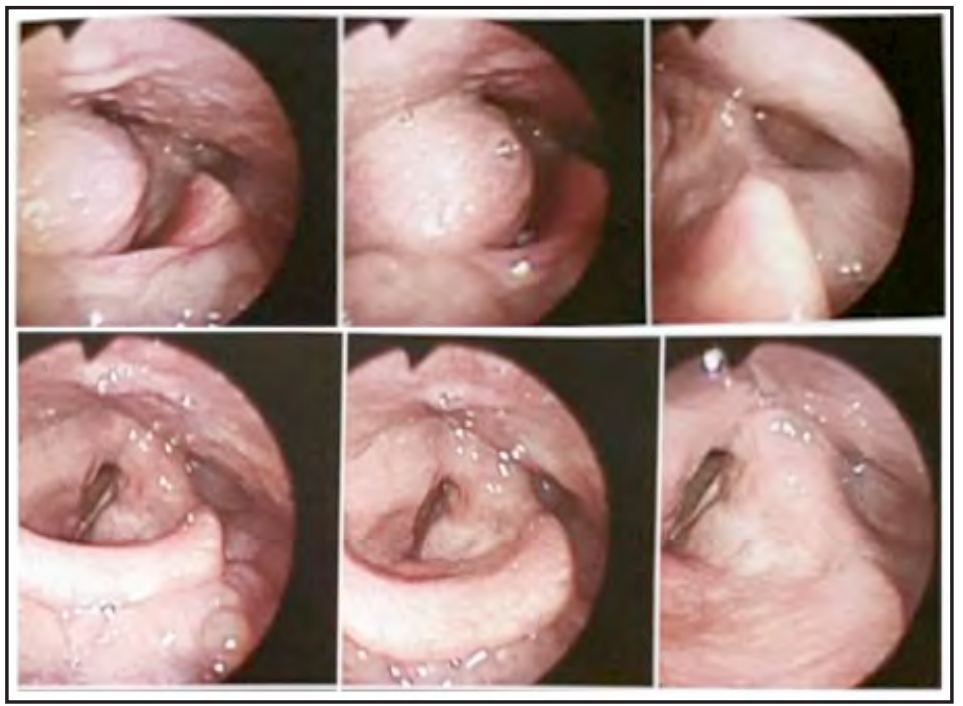

Figura 2. Arriba: nasofaringolaringoscopía preoperatoria. Se observa gran masa de superficie homogénea en receso aritenoepiglótico derecho, con mucosa sana, parcialmente obstructiva. Abajo: Nasofaringolaringoscopía posoperatoria. Se aprecia edema generalizado posquirúrgico, con cuerda vocal izquierda sana, senos piriformes libres.

de la hemilaringe derecha. La cirugía se realizó sin incidentes.

Posteriormente el resultado de la biopsia informó un $\mathrm{HC}$ de laringe. Este diagnóstico fue confirmado por dos patólogos.

El paciente evolucionó de forma favorable, alimentándose de forma inicial por sonda nasogástrica y posteriormente vía oral. Se realizó control con nasofaringolaringoscopía a los 6 días y que mostró edema generalizado posquirúrgico, con cuerda vocal izquierda sana, hipomotilidad de cuerda vocal derecha, senos piriformes libres, con buena sensibilidad supraglótica (Figura 2).

Tras 7 días de hospitalización se decidió decanulación de traqueostomía y fue dado de alta.

\section{DISCUSION}

La Sociedad Internacional para el Estudio de Anomalías Vasculares (ISSVA, por su sigla en inglés) en 1992 estableció una clasificación 
que dividía las anomalías vasculares en dos: 1) Tumores vasculares, donde el hemangioma es el más frecuente, y 2) Malformaciones vasculares, dentro de las cuales, a pesar de su nombre, se encuentra el $\mathrm{HC}$ siendo el más frecuente de esta categoría ${ }^{8}$. Los hemangiomas se caracterizan por tener una fase de proliferación con alta actividad mitótica, con un posterior período de involución. Sin embargo, estos términos han sido utilizados de forma inconsistente en la literatura, por lo que pueden existir errores en cuanto al origen de las lesiones (arterial, capilar o venoso) ${ }^{8}$. A pesar de esto, independiente de si se usa o no la clasificación, los $\mathrm{HC}$ son descritos como lesiones con decoloración azulada $^{2,8-10}$, que en la histología se observan vasos que están ampliamente dilatados, con paredes delgadas, lo que es relativamente constante en la literatura ${ }^{3,7}$. Al parecer la división clínica entre hemangiomas adultos e infantiles es más utilizada y más práctica en cuanto al acercamiento y estudio de estas lesiones, ya que existen diferencias en la presentación de las formas infantiles y adultas. En adultos, es más frecuente en hombres y en la región glótica y supraglótica, a diferencia de los infantiles donde es más frecuente en mujeres y en región subglótica ${ }^{1,3-5}$. En niños las lesiones pueden regresar de forma espontánea, mientras que en los adultos $\mathrm{n} 0^{4,5}$. El caso expuesto, presentaba el perfil más habitual de presentación descrito en la literatura, es decir, un paciente hombre, en la edad media de la vida que se presenta con disfonía progresiva de larga evolución.

Se piensa que como factor etiológico puede estar relacionado el tabaquismo y el trauma larínge $0^{1,6}$. En nuestro caso solo estaba presente el antecedente de tabaquismo.

Por definición, los HC en adultos no pasan por una fase proliferativa, pero pueden crecer en tamaño, en relación a cambios hormonales, infección, trauma o posterior a una cirugía ${ }^{8}$. En general los hemangiomas de adultos no son lesiones muy progresivas, presentándose la mayoría de las veces como cuadros larvados que duran meses 0 años ${ }^{4}$. Cambios patológicos en el tumor, como por ejemplo inflamación, trombosis, hemorragia o cambios quísticos pueden causar crecimiento de la lesión ${ }^{3}$. Ninguno de estos factores pudo ser identificado en nuestro caso.

Si bien la mayoría de las veces se presenta como síntomas no severos y de presentación lar- vada, algunas veces los síntomas progresan hasta llegar al compromiso respiratorio ${ }^{3}$, incluso pueden llevar a la muerte súbita por asfixia, con un mayor riesgo cuando son lesiones pediculadas ${ }^{11}$. Muy rara vez, los hemangiomas se pueden comportar de forma agresiva ${ }^{3}$.

Como se mencionó previamente, el estudio habitualmente se inicia con una nasofaringolaringoscopía donde se pueden observar algunas características que orienten a este tipo de lesión, como es la decoloración azulada o el signo de fonación de Menzel, que consiste en una mayor firmeza, erección, y un color más profundo de la lesión durante la fonación ${ }^{1,2,6}$. El diagnóstico de estas lesiones se puede complementar con una TC, RM 0 ecografía doppler ${ }^{8}$.

En la TC se puede observar como una masa lobulada o multilobulada, isodensa con el músculo que puede infiltrar múltiples espacios profundos del cuello. Se pueden observar "flebolitos", que corresponden a imágenes redondeadas hiperdensas, éstos son considerados por algunos como patognomónicos para malformaciones venosas ${ }^{2,8}$. Además, se puede observar remodelación ósea alrededor de la lesión, lo que es común para malformaciones venosas pero inusual para hemangiomas ${ }^{8}$.

Existe también un reporte de cuatro casos en que se utilizó un cintigrama con glóbulos rojos marcados con tecnecio, lo que podría entregar información anatómica y fisiológica de la lesión ${ }^{2,7}$.

En el caso presentado, el estudio se inició con una TC con contraste de cuello, pero no se encontraban ninguna de las imágenes características de una malformación vascular. La TC junto con la RM puede aportar importante información anatómica, lo que puede ser de gran utilidad para decidir el tratamiento y planificar una intervención quirúrgica. Por otro lado, si bien el cintigrama puede aportarnos información sobre el diagnóstico etiológico, su información anatómica es menos precisa, por lo que no parece útil para la planificación preoperatoria.

Se han utilizado distintas opciones de tratamiento, tales como corticoides sistémicos, corticoides 0 alcohol intralesionales, ablación con láser, interferón, microdebridador, radioterapia, criocirugía 0 cirugía abierta ${ }^{12,5,9,12}$.

La decisión del tratamiento va a depender de la edad, la ubicación, el tamaño y el tipo $0^{1,4}$. En general Ios HC supraglóticos deben ser tratados de forma qui- 
rúrgica cuando son sintomáticos 0 se extienden más allá de la laringe, por lo tanto, es importante descartar previamente el compromiso extralaríngeo al igual que evaluar el tamaño del tumor con imágenes ${ }^{5}$. No existe un procedimiento quirúrgico óptimo universalmente aceptado por lo infrecuente de la patología y la falta de series de $\operatorname{casos}^{5}$. El enfoque habitual es con el acceso mediante faringotomía o laringofisura, sin embargo, esto se reserva para los tumores de mayor tamaño con importante extensión laríngea-hipofaríngea por asociarse a mayor morbilidad intra y posoperatoria ${ }^{5}$.

Posterior al tratamiento los pacientes deben ser controlados en busca de recidivas 5 .

\section{CONCLUSION}

El HC laríngeo en el adulto es una patología muy infrecuente, por lo que no existe un consenso sobre

\section{BIBLIOGRAFIA}

1. Iriz A, Durmaz E, Akmansu SH, Dagli M, Albayrak L, EryiLmaz A. Vocal Cord Hemangioma: A Rare Localization in Adults. Turk J Med Sci 2009; 39 (2): 305-7.

2. Shpitzer T, Noyek A, Witterick I, et al. Noncutaneous Cavernous Hemangiomas of the Head and Neck. Am J Otolaryngol 1997; 18 (6): 367-74.

3. Bridger P, NASSAR V, Skinner H. Hemangioma in the Adult Larynx. Arch Otolaryng 1970; 92: 493-8.

4. Karatayli-Ozgursoy S, Basaran M, Umudum $\mathrm{H}$, Akmansu SH. Adult Laryngeal Hemangioma: A Rare Case Report. Otolaryngology 2015; 5: 201.

5. Lucioni M, Marioni G, Libera D, Rizzotto G. Adult laryngeal hemangioma CO2 laser excision. A single institution 3-year experience. Acta OtoLaryngologica 2006; 126: 621-6.

6. Yilmaz M, Fatma Aktepe F, Altuntas A. Cavernous hemangioma of the left vocal cord. Eur Arch Otorhinolaryngol 2004; 261: 310-11. la mejor opción de tratamiento. El diagnóstico puede sospecharse principalmente frente a un adulto de sexo masculino, en edad media de la vida que se presenta con disfonía o ronquera de meses a años de evolución y que en el estudio se encuentre una masa supraglótica de coloración azulada en la nasofaringolaringoscopía 0 con "flebolitos" en la TC. Es importante la evaluación caso a caso para decidir sobre la mejor opción terapéutica. En lesiones pequeñas, sin extensión extralaríngea existe la opción de observarlas o procedimientos menos invasivos como la cirugía láser, mientras que en grandes lesiones que puedan comprometer la vía aérea, la cirugía abierta mediante abordajes como la laringofisura serían la mejor opción.

Por lo tanto, se necesitan más casos reportados para poder realizar un análisis más acabado y así establecer conductas a seguir según cada caso.

7. Finkelstein M, Noyek A, Kirsh J. Red blood cell scan in cavernous Hemangioma of the larynx. Ann Otol Rhinol Laryngol 1989; 98: 707-12.

8. Baer A, Parmar H, Dipietro M, Kasten S, Mukherul S. Hemangiomas and Vascular Malformations of the Head and Neck: A Simplified Approach. Neuroimag Clin N Am 2011; 21: 641-58.

9. Ibrahimov M, Sari E, Yener M, Karaman E, Enver 0 . Cavernous Hemangioma of the Larynx. The Journal of Craniofacial Surgery 2013; 24: 687.

10. Rodrigues-Pinto E. Cavernous hemangioma of the pharynx and larynx with esophageal involvement. Endoscopy 2015; 47: 213-14.

11. Kıнo L, Byard R. Acute Fatal Upper Airway Obstruction from an Occult Cavernous Hemangioma of the Larynx. J Forensic Sci 2015; 60: 93-6.

12. Yellin S, Labruna A, Anand V. Nd:YaG laser treatment for laryngeal and Hypopharyngeal hemangiomas: a new technique. Ann Otol Rhinol Laryngol 1996; 105: 510-15.

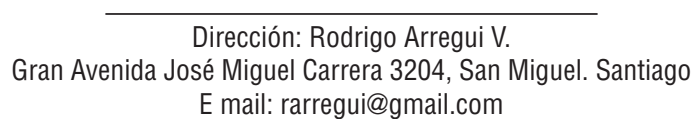

